en ciencias e

Artículo/Article

Sección/Section B

\section{Nuevos casos de leucismo en peces andinos del género Astroblepus (Siluriformes: Astroblepidae) Leucismo en peces andinos}

\section{Fernando Anaguano-Yancha ${ }^{1,2}$}

${ }^{1}$ AmbienConsul, Av. 10 de agosto 695 y Riofrío, Edif, Benalcázar Mil, Ouito, Ecuador. ${ }^{2}$ Dirección actual Wildlife Conservation Society, Ecuador Program, Quito 170501, Ecuador

Autor para correspondencia/Corresponding autor, e-mail. fernando_anaguano@hotmail com

\section{New records of leucism in Andean fish of the genus Astroblepus (Siluriformes: Astroblepidae)}

\section{Abstract}

The South American ichthyofauna is the most diverse in the world, however, it is the least studied. For example, there are few reports of pigmentation anomaly in freshwater fishes, especially in high Andean species. This study reports two new cases of leucism in two species of the genus Astroblepus (Siluriformes: Astroblepidae). The individuals presented depigmentation in all body, except in the ocular iris, caudal peduncle, dorsal and caudal fins. Possibly, inbreeding and habitats contamination are causing stress in these fish populations, enhacing the appearance of this aberration.

Keywords: Freshwater fishes, Siluriformes, Pigmentation anomaly, Contamination of

\section{Resumen}

La ictiofauna Sudamericana es la más diversa del mundo, sin embargo, es también la menos estudiada. Por ejemplo, son escasos los reportes de casos de aberraciones pigmentarias en peces dulceacuícolas, especialmente en especies altoandinas. Este estudio reporta dos nuevos casos de leucismo en dos especies del género Astroblepus (Siluriformes: Astroblepidae). Los individuos presentaron despigmentación en casi todo el cuerpo, excepto en el iris ocular, pedúnculo caudal aletas dorsales y caudales. Posiblemente, la endogamia y la contaminación de sus hábitats estén causando estrés en estas poblaciones de peces, potenciado la aparición de esta aberración.

Palabras clave: Peces dulceacuícolas, Siluriformes, Aberraciones pigmentarias, Contaminación de hábitats.
La coloración tegumentaria en los peces es producida por los cromatóforos, que incluyen a los melanóforos, xantóforos, eritróforos, leucóforos e iridóforos [1]. La carencia de pigmento tegumentario causa coloraciones anormales en los peces, y de acuerdo con las características fenotípicas de los individuos, se denominan albinismo, leucismo, melanismo, xantismo, ambicoloración, metacromismo y policromismo [2-5]. El leucismo es atribuido a la mutación de genes recesivos en el desarrollo embrionario, lo cual, inhibe la migración de melanoblastos (células productoras de pigmentos) de la cresta neural a la capa basal de la epidermis $[6,7]$. Generalmente se manifiesta en los organismos por la ausencia total o parcial de la pigmentación en la piel, pero una pigmentación normal de las retinas [8-10]. Sin embargo, en muchos casos, la etiología de las aberraciones cromáticas no está clara y varios factores, incluyendo mutaciones genéticas no patológicas, aislamiento poblacional, estrés ambiental y la exposición a metales pesados pueden estar implicados en el surgimiento de estas anomalías [11-13].

En peces dulceacuícolas sudamericanos se ha reportado casos de aberraciones pigmentarias en especies de las familias Erythrinidae, Lebiasinidae, Doradidae, Pimelodidae, Callichthyidae, Loricariidae, Atroblepidae, Gymnotidae y Lepidosirenidae [14-17]. Entre las especies de la familia Astroblepidae, un grupo de pequeños bagres neotrópicales, se han reportado ocurrencia de leucismo en Astroblepus ubidiai procedente de una población aisladas de la cuenca del río Mira y en Astroblepus pholeter que habitan la caverna de Latas, Cuenca del río Napo [15, 16]. El objetivo del presente estudio es reportar casos de leucismo en Astroblepus fissidens y Astroblepus brachycephalus. Los ejemplares leucísticos provienen de metapoblaciones, que habitan cuerpos de agua en la región Andina y Litoral de Ecuador (Fig. 1).

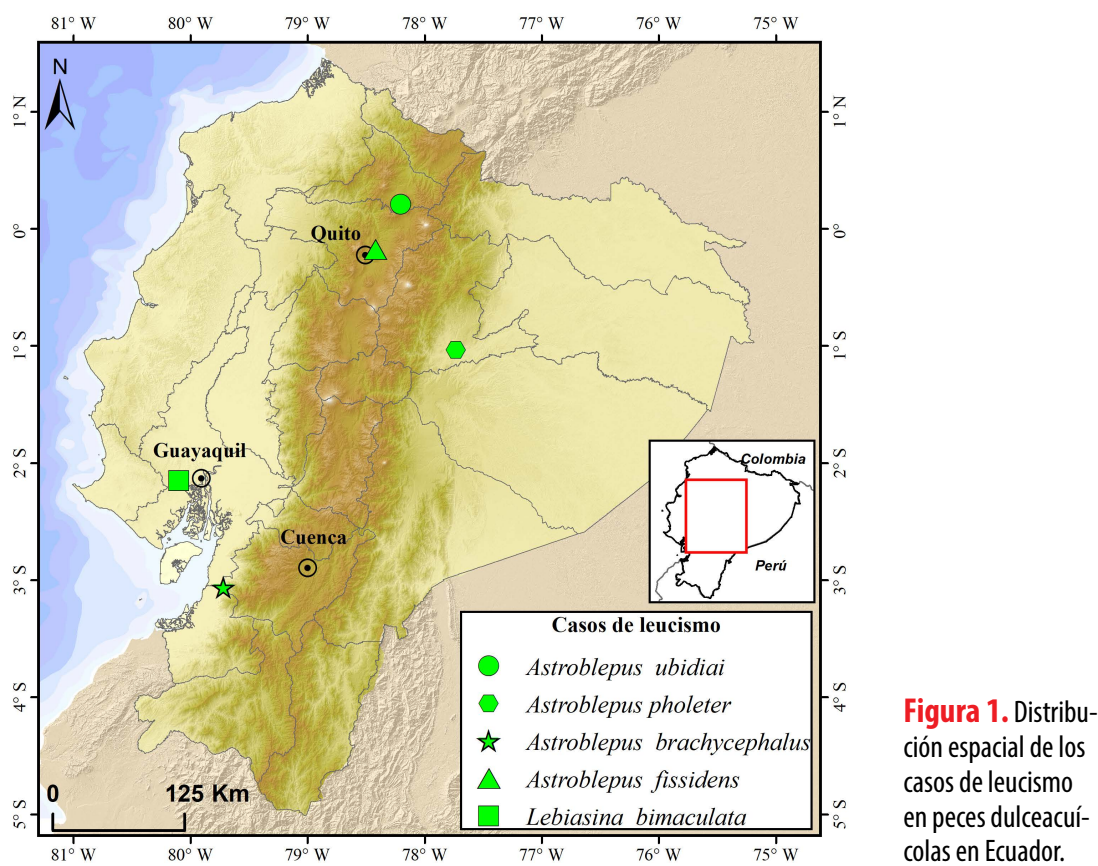


Un macho adulto de $A$. fissidens de $70,23 \mathrm{~mm}$ de longitud estándar con leucismo fue fotografiado (cámara SONY DSC-W830) en la vertiente Amabulo, afluente del río San Pedro, parroquia Nayón, cantón Quito, provincia de Pichincha, cuenca alta del río Esmeraldas, $\quad(-0.175447 \mathrm{~S}-78.418791 \mathrm{~W}, 2100 \mathrm{~m})$, el 28 de febrero 2012. Un macho juvenil de $A$. brachycephalus de $40,75 \mathrm{~mm}$ de longitud estándar con leucismo fue colectado en el río Villa, cantón Ponce Enríquez, provincia de Azuay, cuenca del río Siete (-3.0632015-79.719691W de $A$. fissidens y su evidencia se está registrada en archivos fotográficos. El espécimen de $A$. brachycephalus se encuentran depositados en el Museo Ecuatoriano de Ciencias Naturales, Instituto Nacional de Biodiversidad (MECN-DP3528).

Los individuos de $A$. fissidens y $A$. brachycephalus generalmente presentan un variado patrón de coloración, típicamente presentan dorsos de color marrón, pardo obscuro o gris, con manchas pequeñas de diversas tonalidades de negro, marrón y blanco. Las aletas pueden presentar los mismos colores y patrones mencionados para el dorso o pueden ser amarillas (Figs. 2A, C). Los ejemplares leucísticos presentaron despigmentación en todo el cuerpo, excepto en el pedúnculo, parte superior de la cabeza y en los radios duros de la aleta dorsal y caudal, donde observamos pequeñas manchas oscuras; en ambos casos la coloración de la piel es rosada-rojiza (Figs. 2B, D).

$2 A$.

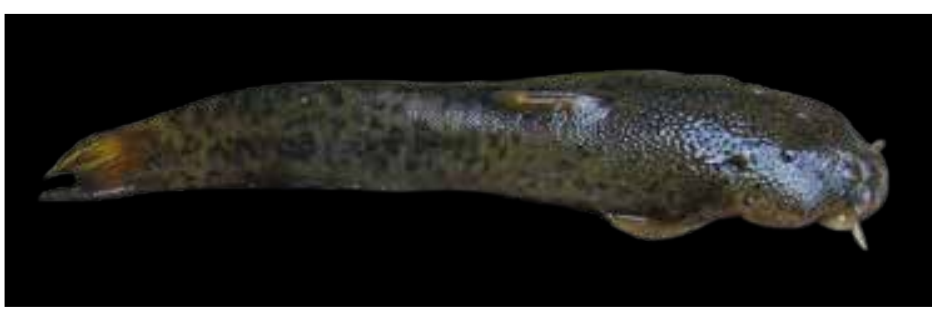

2B.

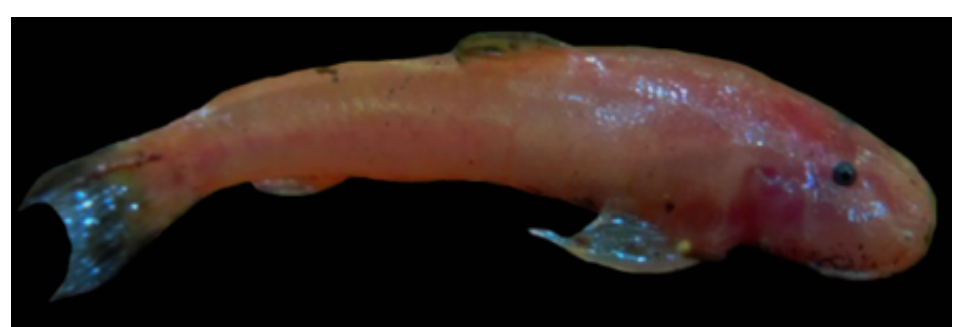

2C.

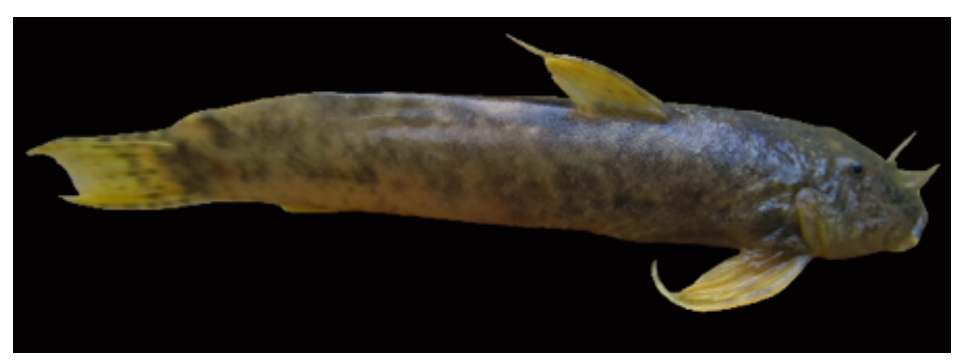

2D.

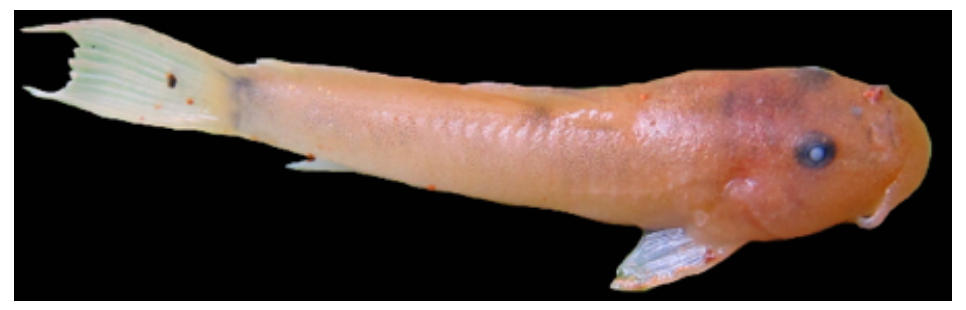

Figura 2. Patrón de coloración típicos y leucísticos: A). Ejemplar de Astroblepus fissidens con coloración normal $(98 \mathrm{~mm})$; B). Ejemplar de Astroblepus fissidens leucistico $(70,23 \mathrm{~mm})$; C) Ejemplar de Astroblepus brachycephalus con coloración normal (94,34mm); D). Ejemplar de Astroblepus brachycephaIus leucistico (40,75mm). Fotografías: F. Anaguano-Yancha.

A pesar de la enorme diversidad de especies de peces presentes en Sudamérica, los reportes de aberraciones pigmentarias en la ictiofauna son muy escasos. Actualmente existen 20 casos reportados en 18 especies. Ocho casos se han reportado en Brasil, cuatro en Argentina, tres en Ecuador, un caso en Uruguay y tres casos no proporcionar referencias sobre la localidad, el $70 \%$ de estos casos corresponden albinismo y $30 \%$ a leucismo [Tabla 1]. En Ecuador son recientes los reportes de leucismo en vertebrados, atribuidos principalmente a la alta probabilidad de endogamia a causa del aislamiento poblacional $[15,30]$. Además, recientemente se ha corroborado la reducción de pigmentación en astroblépidos que habitan galerías subterráneas [16].

A nivel global, los ecosistemas de agua dulce son los más amenazados, principalmente, por el crecimiento de la población humana y el desarrollo económico [31, 32]. Las condiciones físicas y químicas del agua son frecuentemente modificadas por el incremento de sedimentos en el agua la remoción del sustrato, la reducción del volumen de agua, la construcción de presas y las descargas de vertimientos residuales sin tratamiento previo, provenientes de la industria y de las cloacas de ciudades y poblados $[33,34]$. Estas afectaciones modifican la estructura y conexión de los sistemas acuáticos dentro de la red hídrica ocasionando el aislamiento poblacional, interrumpiendo el flujo génico [34]. Sumado a esto, la contaminación por metales pesados causa efectos importantes en las condiciones de salud de los peces, así como también afecciones genéticas [12, 35].

Varios estudios han expuesto que las altas frecuencias de aberraciones pigmentarias en poblaciones de fauna silvestre podrían ser indicativos de aislamiento poblacional, contaminación, exposición a metales pesados o estrés ambiental [11-13, 27]. Durante varias décadas, los ríos San Pedroy Siete han sido contaminados por descargas industriales y domésticas de las ciudades y poblados aledaños. Análisis de sus aguas y sedimentos indican que hay un grado de perturbación antropogénico muy severo [36, 37], lo que ha provocado que las poblaciones de $A$. fissidens y $A$. brachycephalus queden aisladas en pequeños esteros sin conexión hacia la cuenca principal, reduciéndose el flujo genético, cual lo reportado para ecosistemas acuáticos en los Andes Tropicales que presentan un alto grado de contaminación [34]. Probablemente el aislamiento poblacional, aumente la ocurrencia de endogamia propiciando la manifestación de aberraciones cromáticas. Sin embargo, no se puede aseverar que esta sea la causa, razón por la cual, es necesario realizar estudios futuros sobre la variabilidad genética de estas especies en las cuencas antes mencionada para probar esta hipótesis. 
210

Otra posible explicación de la manifestación de aberraciones cromáticas en $A$. brachycephalus podría estar relacionada con desordenes genéticos causados por la exposición a metales pesados, como los evidenciados en arroyos que sufren contaminación de sus sustratos por Cadmio (Cd) y Cromo (Cr) en Brasil [12]. La extracción de oro constituye una fuente importante de contaminación por metales pesados de los ríos que drenan las áreas mineras del cantón Camilo Ponce Enríquez, donde se ha reportado altas concentraciones de metales pesados en sus sedimentos, especialmente de Cd [37], que supera los estándares de calidad de los sedimentos establecidos por el Ministerio del Ambiente de Canadá para la protección de la fauna acuática [38]. A pesar de esto, no se puede aseverar que esta sea la causa, ya que se ha reportado alto contenidos de metales pesados en peces bentónicos [37], así como también en aves cual, es indispensable realizar una investigación más exhaustiva de estos aspectos en las concesiones mineras del Ecuador, para determinar si la exposición a metales pesados propicia la manifestación de aberraciones cromáticas en la fauna silvestre.

Figura 3. Hábitats de los registros de leucismo: A) Vertiente Amabulo; B) Río San Pedro, nótese el alto grado de contaminación; C) Río Villa ; D) Actividad minera en el río Villa. Fotografías: J. Juiña (A), F. Anaguano-Yancha $(C, B)$, T. Meza (D).

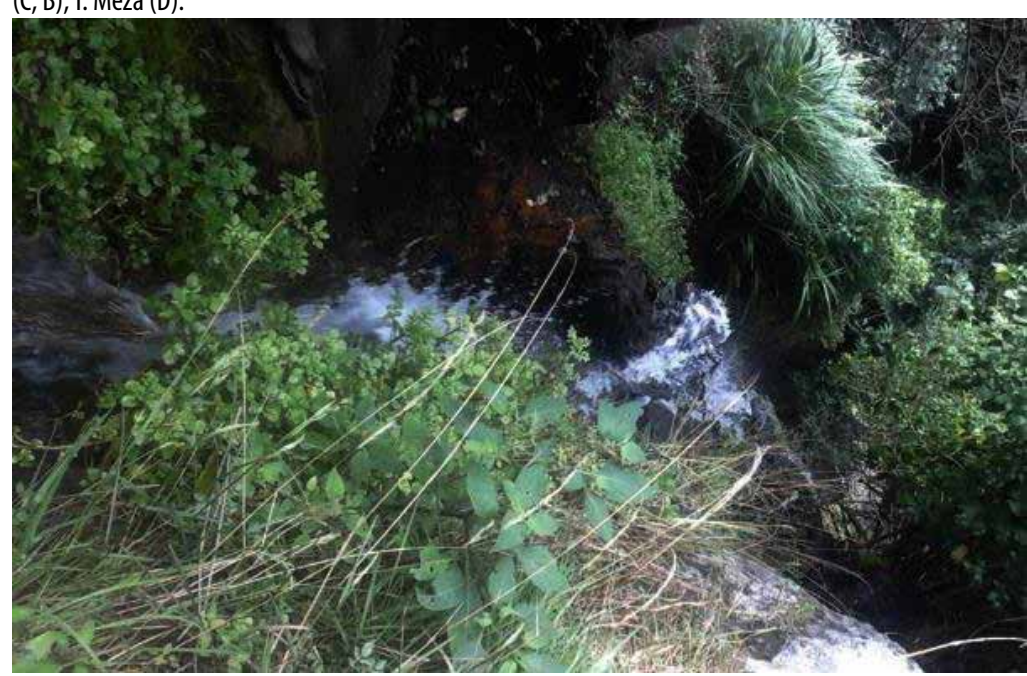

3A.

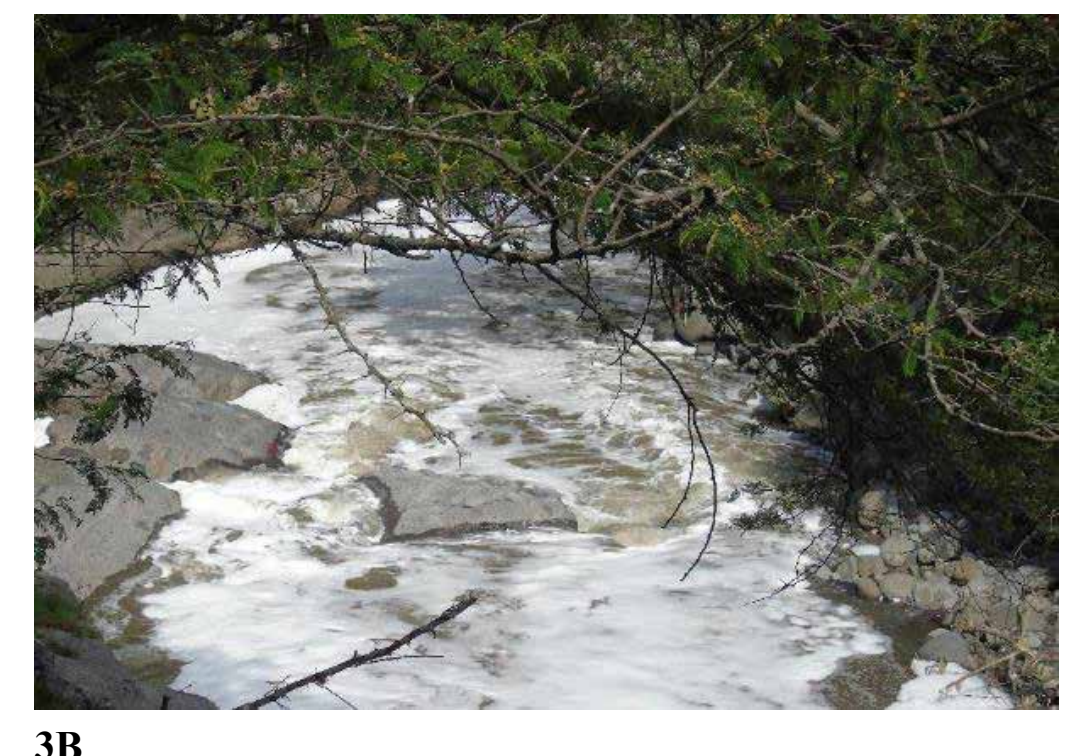

3B

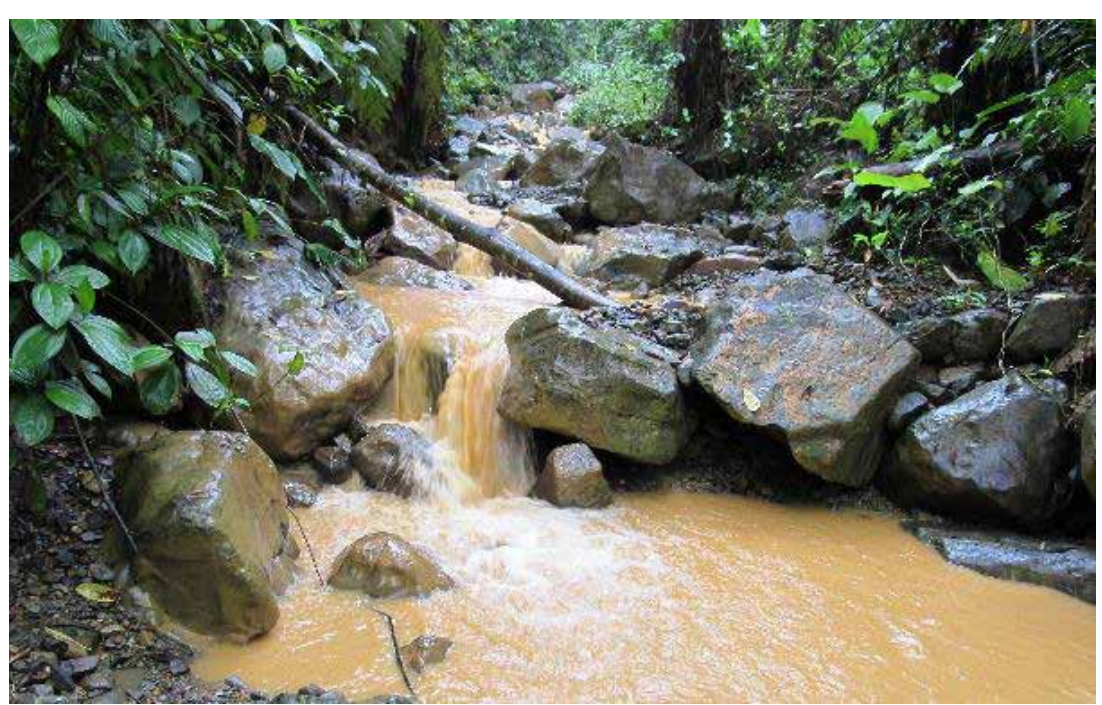

3C. 


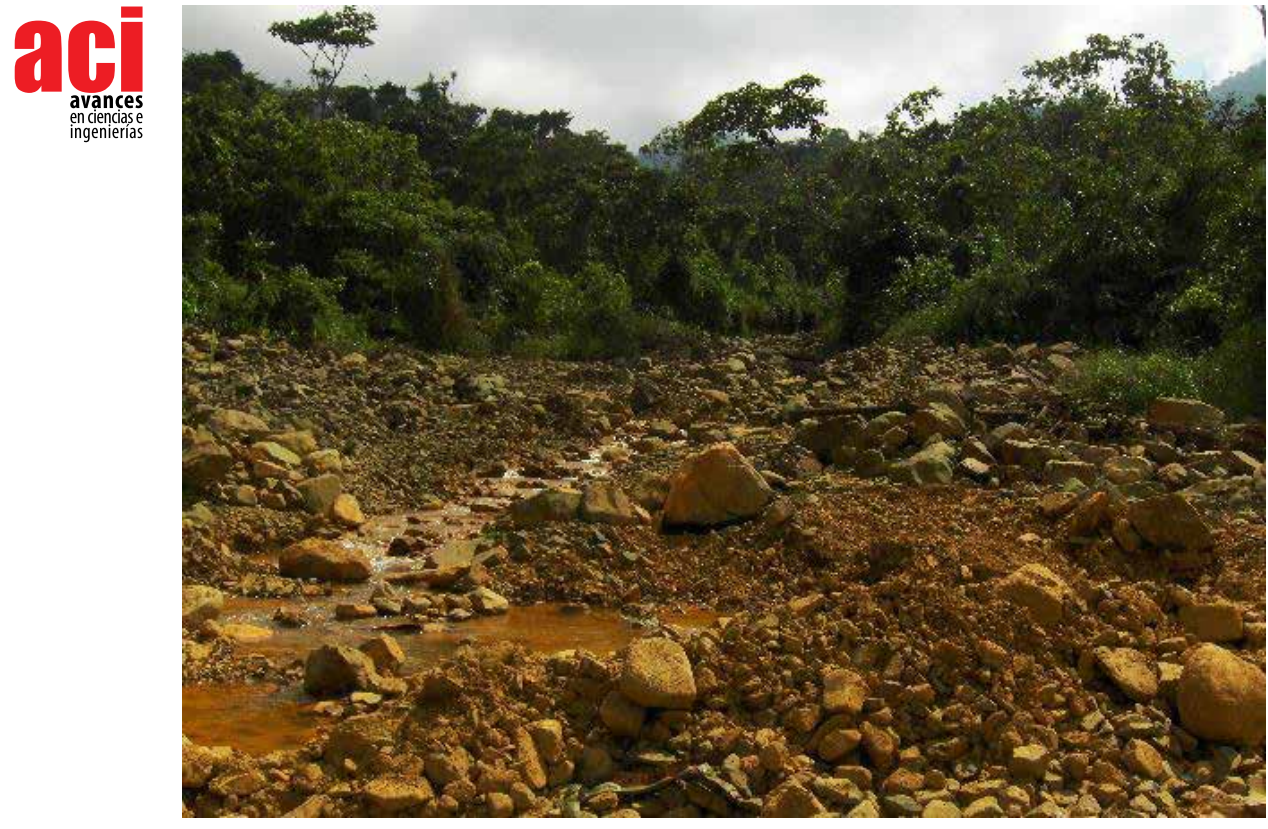

$3 D$.
Tabla 1. Aberraciones cromáticas reportadas para Sudamérica en peces de agua dulce.

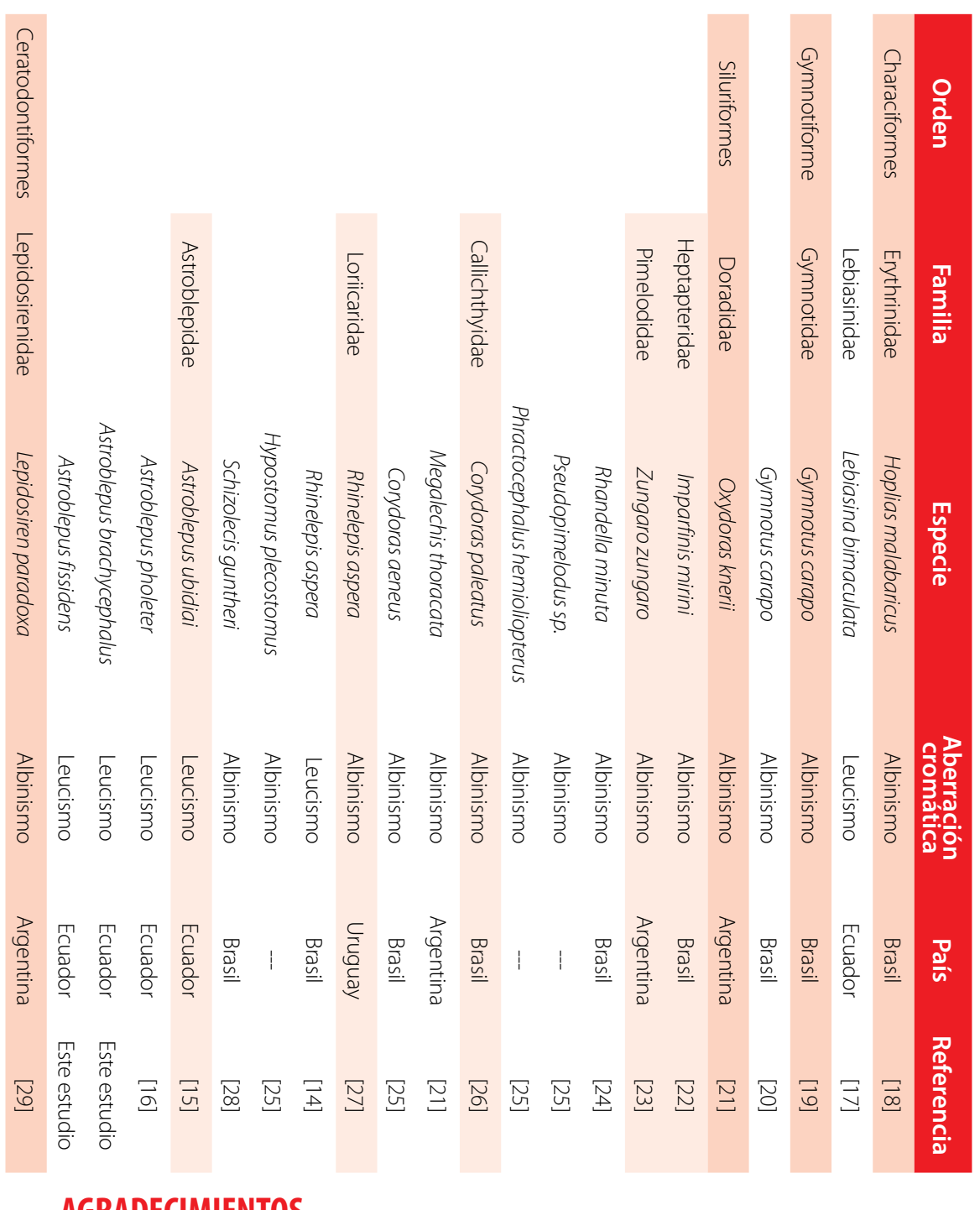

AGRADECIMIENTOS

Agradezco a la Consultora socio ambiental AmbienConsul, que contrató mis servicios para la realización de evaluaciones faunísticas en varios puntos del proyecto minero Gaby-Papagrande (provincia de Azuay), en el marco del desarrollo de los estudios socioambientales que se realizaron en la zona. A Naveda, J. Brito, D. Racines y dos revisores anónimos revisaron el manuscrito, realizando valiosos aportes. La Dirección Provincial del Ambiente del Azuay otorgó la autorización de investigación № 019-AZ-2011. 


\section{0}

\section{REFERENCIAS}

[1] Fujii, R. (2000). The regulation of motile activity in fish chromatophores. Pigment Cell Research, 13(5), 300-319. doi: Fujii, R. (2000). The regulation of motile activity in fish chrom
http://www.dx.doi.org/10.1034/j.1600-0749.2000.130502.x

[2] Dawson, C. (1964). A Bibliography of Anomalies of Fishes. Gulf Research Reports, 1 (6), 308-399. doi: http://www. dx.doi.org/10.18785/grr.0106.01

[3] Dawson, C. (1966). A Bibliography of Anomalies of Fishes, Supplement 1. Gulf Research Reports, 2 (2), 169-176. doi: http://www.dx.doi.org/10.18785/grr.0202.03

[4] Dawson, C. (1971). A Bibliography of Anomalies of Fishes, Supplement 2. Gulf Research Reports, 3 (2): 215-239. doi: http://www.dx.doi.org/10.18785/grr.0302.05

[5] Dawson, C. \& Heal, E. (1976). A Bibliography of Anomalies of Fishes: Supplement 3. Gulf Research Reports, 5 (2), 35-41. doi: http://www.dx.doi.org/10.18785/grr.0502.05

[6] Shin, M. K. (2000). Controlling Gene Expression in Mice with Tetracycline: Application in Pigment Cell Research. Pigment Cell Research, 13, 326-331. Recuperado de: https://onlinelibrary.wiley.com/doi/pdf/10.1034/j.1600749.2000.130504.x

[7] Sugimoto, M. (2002). Morphological Color Changes in Fish: Regulation of Pigment Cell Density and Morphology. Microscopy Research and Technique, 58, 496-503. doi: https://doi/pdf/10.1002/jemt.10168

[8] Clark, S. (2002). First Report of Albinism in the White- Spotted Bamboo Shark, Chiloscyllium plagiosum (Orectolobiformes: Hemiscyllidae), With a Review of Reported Color Aberrations in Elasmobranchs. Zoo Biology, 21, 519-524. doi: https://doi.org/10.1002/zo0.10068

[9] Evangelista-Leal, M., Horst-Schulz, U., Lehmann-Albornoz, P., Machado, R., \& Ott, P. H. (2013). First record of partiad albinism in two cattish species of Genidens (Siluriformes: Ariidae) in an estuary of Southern Brazil. Brazilian Archives of Biology and Technology, 56(2), 237-240. Recuperado de: http://www.scielo.br/pdf//babt/156n2/08.pdf

[10] Bigman, J. S., Knuckey, J. . . S. \& Ebert, D. (2015). Color aberrations in Chondrichthyan fishes: first records in the genus Bathyraja (Chondrichthyes: Rajiformes: Arhynchobatidae). Marine Biodiversity, 46(3), 579-587. doi: http://www. Bathyraja (Chondrichthyes: Rajformes: $A$ -
dx.doi.org/10.1007/s12526-015-0403-z

[11] Bensch, S., Hansson, B., Hasselquist, D., \& Nielsen, B. (2000). Partial albinism in a semi-isolated population of great reed warblers. Hereditas, 133, 167-170. doi: https://doi.org/10.1111/j.1601-5223.2000.t01-1-00167.x

[12] Baggio, H., \& Horn, A. H. (2010). Teores totais e distribuic, ão dos metais pesados $\mathrm{Cu}$, Cd, Cr, Ni Pb e Zn nos sedimentos de corrente e fundo do rio do Formoso- MG. Geografias, 6, 107-117. Recuperado de: http://www.igc.ufmg br/ portaldeperiodicos/index.php/geografias/article/view/504/375.

[13] Quigley, D. T. G., Lord, R., MacGabhann, D., \& Flannery, K. (2017). First records of xanthochromism in three-bearded rockling Gaidropsarus vulgaris (Cloquet, 1824) and pollack Pollachius pollachius (Linnaeus, 1758). Journal Applied of Ichthyology, 33(6), 1208-1210. doi: https://doi.org/10.1111//jai.13456

[14] Nobile, A. B., Freitas-Souza, D., de Lima, F. P., Acosta, A., \& Da Silva, R. J. (2016). Partial albinism in Rhinelepis aspera from the Upper Paraná Basin, Brazil, with a review of albinism in South American freshwater fishes. Revista Mexican de Biodiversidad, 87 (2), 531-534. doi: http://www.dx.doi.org/10.1016/j.rmb.2016.04.005

[15] Mena-Valenzuela, P., \& Valdiviezo-Rivera, J. (2016). Leucismo en Astroblepus ubidiai (Pellegrin 1931) (Siluriformes: Astroblepidae), de la provincia de Imbabura, Ecuador. Biota Colombiana, 17 (1), 131-136. doi: http://www.dx.doi. Astroblepidae), de la provincia de
org/10.21068/(2016v17r01a10

[16] Espinasa, L., Robinson, J., Soares, D., Hoese, G., Toulkeridis, T. \& Toomey, T. (2018). Troglomorphic features of Astroblepus pholeter, a cavefish from Ecuador, and possible introgressive hybridization. Subterranean Biology,27, 17-29. doi:

[17] Nugra, F., Anaguano-Yancha, F., Arízaga, C., Zarate, E. \& Brito, J (En prensa). Leucismo en el pez Lebiasina bimaculate (Characiformes: Lebiasinidae) en Guayas, Ecuador. Biotacolombiana, 19(2)

[18] Silva, T., Araújo, T.\& Bicudo, A. (2013). First report of albinism in trahira Hoplias malabaricus from Brazil. Boletim do Instituto de Pesca de São Paulo, 39, 457-460. https://www.pesca.agricultura.sp.gov.br/39_4_457-460.pdf

[19] Campos-da Paz, R. \& Caramaschi, E. P. (1994). First record of albinism in a gymnotiform fish (Teleostei: Ostariophysi). Ichthyological Exploration of Freshwaters, 5, 1-4.

[20] Oliveira, C. \& Foresti, F. (1996). Albinism in the banded knifefish, Gymnotus carapo. Tropical Fish Hobbyist, 44, 92-96.

[21] Panattieri, A. E. \& Del Barco, D. M. (1980). Un caso de albinismo en armado chancho Oxidoras kneri Bleeker 1862 Pisces, Doradidae. Comunicaciones del Museo Provincial de Ciencias Naturales Florentino Ameghino, 9, 9-10.
[22] Manoel, P., Ono, E. \& Alves, I. (2017). First report of albinism in the South American catfish Imparfinis mirini (Siluriformes: Heptapteridae). Revista Mexicana de Biodiversidad, 88(2), 471-473. https://www.sciencedirect.com/ science/article/pii/S1870345317300799

[23] Taberner, R., Fernández-Santos, J. \& Castelli, J. (1976). Un manguruyú albino Paulicea luetkeni (Steindachner 1876) Eigenmann 1910. Physis B, 35, 121-123.

[24] Sazima, I. y Pombal, J. P. (1986). Um albino de Rhamdella minuta, com notas sobre comportamento (0steichthyes, Pimelodidae). Revista Brasileira de Biologia, 46, 377-381. https://www.researchgate.net/publication/277776337_ Um_albino_de_Rhamdella_minuta_com_notas_sobre_comportamento_0steichthyes_Pimelodidae

[25] Burgess, W. E. (1989). An atlas of freshwater and marine catfishes: a preliminary survey of the Siluriformes. New Jersey: TFH Publications. $784 \mathrm{pp}$.

[26] Fach, D. (1963). Albinotische Corydoras paleatus. Aquarien Terrarien Zeitschriften, 16, 225-227.

[27] Luengo, J. A. (1965). Un caso de albinismo en Rhinelepis aspera Agassiz del Uruguay (Pisces, Loricariidae). Neotropica, $11,51-52$.

[28] Brito, M. F. G. \& Caramaschi, E.P. (2005). An albino armored catfish Schizolecis guntheri (Siluriformes: Loricariidae) from an Atlantic Forest coastal basin. Neotropical lchthyology, 3, 123-125. http://www.scielo.br/pdf/ni//v3n1/v3n1a09.pdf http://www.dx.doi.org/10.3897/subtbiol.27.2709

[29] Azpelicueta, M. M. \& Braga, L. (1984). Albinismo en Lepidosiren paradoxa Fitzinger, 1837 (Osteichthyes, Dipnoi, Lepidosirenidae). Neotropica, 30, 75-78.

[30] [16] Brito, J., \& Valdivieso-Bermeo, K. (2016). First records of leucism in eight species of small mammals (Mammalia: Rodentia). Therya, 7, 483-489. doi: http://www.dx.doi.org/10.12933/therya-16408

[31] Russi, D., Brink, P., Farmer, A., Badura, T., Coates, D., Förster, J., Kumar, R., \& Davidson, N. (2013). The Economics of Ecosystems and Biodiversity for Water and Wetlands. London and Brussels: Institute for European Environmental Policy; Gland: Ramsar Secretariat. Recuperado de: https://www.ramsar.org/sites/default/files/documents/library/ teeb_waterwetlands_report_2013.pdf

[32] Vörösmarty, C.J., Mclntyre, P.B., Gessner, M.O., Dudgeon, D., Prusevich, A., Green, P., Glidden, S., Bunn, S.E., Sullivan, C.A., Liermann, C.R., \& Davies, P.M. (2010). Global threats to human water security and river biodiversity. Nature, 467, 555-561. doi: http://www.dx.doi.org/10.1038/nature09440 10.1038/nature09440

[33] Dudgeon, D. (2012). Threats to freshwater biodiversity globally and in the Indo-Burma Biodiversity Hotspot. En D. J. Allen, K. G. Smith, \& W. R. T. Darwall (Eds.), The Status and Distribution of Freshwater Biodiversity in Indo-Burma, pp. 1-25. Cambridge and Gland, Switzerland: IUCN. Recuperado de: https://www.iucn.org/sites/dev/files/import/ downloads /indo_burma_report_complete_low_res_28_aug_1.pdf

[34] Tognelli, M.F., Lasso, C.A., Bota-Sierra, C.A., Jimenez-Segura, L.F. \& Cox, N.A. (Eds.). (2016). Estado de Conservación y Distribución de la Biodiversidad de Agua Dulce en los Andes Tropicales. Gland, Cambridge and Arlington: UICN. doi: http://dx.doi.org/10.2305/UCN.CH.2016.02.es

[35] Palacio, J.A. (2007). Ecotoxicología acuática. Medellín: Editorial Universidad de Antioquia.

[36] Campaña, A., Gualoto, E., \& Chiluisa-Utreras, V. (2017). Evaluación fisico-química y microbiológica de la calidad del agua de los ríos Machángara y Monjas de la red hídrica del distrito metropolitano de Quito. Bionatura, 2(2), 305-310. doi: http://www.dx.doi.org/10.21931/RB/2017.02.02.6

[37] Tarras-Wahlberg, N. H., Flachier, A., Fredriksson, G., Lane, S., Lundberg, B., \& Sangfors, 0. (2000). Environmental Impact of Small-scale and Artisanal Gold Mining in Southern Ecuador: Implications for the Setting of Environmental . org/10.1579/0044-7447-29.8.484

38] Canadian Council of Ministers of the Environment. (2001). Canadian sediment quality guidelines for the protection of aquatic life: Summary tables. Updated. En Canadian Environmental Quality Guidelines, 1999. Winnipeg: Canadian Council of Ministers of the Environment. Recuperado de: https://www.elaw.org/system/files/sediment_summary_table.pdf

[39] Albuja, L., Montalvo, L., Cáceres, F., \& Jácome, N. (2011). Niveles de mercurio en aves silvestres de tres regiones mineras del sur del Ecuador. Revista Politécnica, 30(3), 18-32. Recuperado de: http://bibdigital.epn.edu.ec/ bitstream/15000/5239/1/Mercurio-Aves-Ecuador\%202012Politecnica30(3).pdf 